\title{
Tissue Expression of Methyltransferases in Response to Acute and Long-term Exercise in Sedentary Men
}

Thomas Olsen ( $\sim$ thomas.olsen@medisin.uio.no)

Department of Nutrition, Institute of Basic Medical Sciences, Faculty of Medicine, University of Oslo, Sognsvannveien 9, 0317 Oslo, Norway

Sindre Lee

University of Oslo

Kathrine Vinknes

University of Oslo

Frode Norheim

University of Oslo

Marit Hjorth

University of Oslo

Cheryl Turner

University of Oxford

Helga Refsum

University of Oslo

Jørgen Jensen

Norwegian School of Sport Sciences

Kåre Birkeland

University of Oslo

Christian Drevon

University of Oslo

\section{Research Article}

Keywords: Exercise influences epigenetic regulation, acute exercise (AE), tissue methyltransferase activity

Posted Date: January 14th, 2021

DOI: https://doi.org/10.21203/rs.3.rs-143201/v1

License: (c) (i) This work is licensed under a Creative Commons Attribution 4.0 International License.

Read Full License 


\section{Abstract}

Exercise influences epigenetic regulation of gene expression by modulating tissue methyltransferase activity, whereas effects on methyltransferases with other crucial biological functions have not been elucidated. We performed RNA sequencing of skeletal muscle (SkM) and white adipose tissue (WAT) obtained from 26 sedentary men undergoing acute exercise (AE) and a long-term, 12-week exercise intervention (LTE). We investigated exercise effects on tissue methyltransferase transcripts and a plasma marker of methylation capacity (methionine/homocysteine ratio). Blood and tissue samples were obtained before, just after and $2 \mathrm{~h}$ after AE (blood and SkM), and before and after LTE (blood, SkM, WAT). Differential expression analyses revealed that 43 (15 up; 26 down) and 55 (31 up; 23 down) methyltransferases were differentially expressed in SkM just after and $2 \mathrm{~h}$ after $\mathrm{AE}$, respectively. After LTE, 69 methyltransferases (13 up; 55 down) were differentially expressed in SkM. Upregulated methyltransferases were implicated in histone and peptidyl-lysine methylation ( $A E, 0 \mathrm{~h}$ ), RNA processing ( $A E, 2 \mathrm{~h}$ ), and cell communication (LTE). Downregulated methyltransferases were implicated in gene expression ( $\mathrm{AE}, \mathrm{O} \mathrm{h}$ ) and $\mathrm{mRNA}$ processing (LTE). Plasma methionine/homocysteine decreased after $\mathrm{AE}$, but was elevated after LTE. In conclusion, AE and LTE influence SkM but not WAT methyltransferase transcript levels and plasma methionine/homocysteine.

\section{Introduction}

Methyltransferases comprise a heterogeneous family of more than 200 enzymes catalyzing the transfer of methyl groups from methyl donors to methyl acceptors including DNA, RNA, histones, proteins, and other molecules ${ }^{1}$. Methylation reactions are crucial for several biologic processes including epigenetic regulation of gene expression, post-translational modification of proteins, signaling and biosynthesis. Methyl groups are derived from the universal methyl donor, S-adenosylmethionine (SAM), an intermediate in the metabolism of the essential sulfur-containing amino acid methionine ${ }^{2}$, ultimately yielding a methylated product and homocysteine (transmethylation). Aberrant methylation patterns and plasma markers of methylation reactions have been associated with progression of metabolic diseases including obesity, cardiovascular disease, and type 2 diabetes mellitus ${ }^{3-9}$.

Physical activity is beneficial for prevention and treatment of metabolic diseases and some forms of cancer ${ }^{10}$. In humans, a particular area of focus in exercise intervention trials has been to investigate the effects of exercise on DNA methylation in skeletal muscle (SkM) with a focus on epigenetic regulation in SkM adaptations to exercise ${ }^{11-16}$. Considerably less effort has been dedicated SkM methyltransferases in other processes despite some targeted studies demonstrating or suggesting additional roles in signaling ${ }^{17}$, tissue remodeling ${ }^{18}$ and post-translational modifications ${ }^{19}$ in response to either acute or long-term exercise. Indeed, current evidence implies that the link between methylation reactions and exercise can be extended beyond DNA methylation, but no systematic investigations have been published on this topic. 
We used data from an exercise intervention on 26 sedentary men to investigate the transcriptomic response of genes encoding proteins with established or probable methyltransferase activities, or known to be components of enzyme complexes with methyltransferase activities, to acute exercise, after $2 \mathrm{~h}$ of rest (SkM) and to long-term exercise intervention (SkM and subcutaneous white adipose tissue [WAT]). Next, using gene set enrichment analyses we investigated gene ontology (GO) biologic processes associated with upregulated and downregulated methyltransferase genes. Because exercise induce dramatic decreases in plasma methionine ${ }^{20,21}$ which may directly influence intracellular SAM availability for methyltransferase reactions ${ }^{22-25}$, we evaluated the effects of acute and long-term exercise on a plasma marker of methylation capacity (Methionine/Homocysteine [Met/Hcy]), which is considered a potential indicator of SAM-dependent methylation reactions in the cell ${ }^{7,8}$. Finally, we explored the potential links between exercise-induced changes in methyltransferase transcripts with changes in indicators of metabolic health including insulin sensitivity, $\mathrm{VO}_{2} \mathrm{max}$, muscle hypertrophy and body fat after the long-term exercise intervention.

\section{Results}

\section{Study participants}

Subject characteristics and main outcome data that have been published previously ${ }^{26}$. A presentation of the study subjects included in the present study can be found in Supplementary Table 1. In brief, the main findings of the exercise intervention were that sujects increased $\mathrm{GIR}, \mathrm{VO}_{2} \max$, chest press, pull-down and leg press strength, as well as thigh cross-sectional area.

\section{Effects of acute exercise on methyltransferase mRNAs in SkM and WAT}

Average differential expression of methyltransferases across the two acute exercise bouts are shown in Figs. 1-2 and Supplementary Tables 2-3. Just after exercise, a total of 15 transcripts were increased whereas 26 transcripts were decreased (Fig. 1A). The top 5 most upregulated methyltransferases were $N N M T$, IRF4, RRP8, MEPCE, and NCOA6, whereas the top 5 most downregulated were MECOM, PRMT6, METTL18, BCDIN3D, and EHMT2.

After $2 \mathrm{~h}$ rest, 31 mRNA transcripts were increased, whereas 23 were decreased (Fig. 2A and Supplementary Table 3). NNMT and RRP8 remained upregulated, whereas IRF4, MEPCE, and NCOA6 had returned to baseline levels. Other upregulated methyltransferases were METTL21C, SETDB2, and $M E T T L 7 B$. Of the top 5 most downregulated methyltransferases just after acute exercise, only EHMT2 remained downregulated after $2 \mathrm{~h}$ rest. Significantly downregulated methyltransferases after $2 \mathrm{~h}$ rest otherwise included HDAC9, DNMT3B, INMT, SETD1B, and TARBP1. We did not observe group differences in differential expression after acute exercise in SkM. 
In total, 37 out of 210 methyltransferases were upregulated after acute exercise. The majority were increased only after $2 \mathrm{~h}$ rest, whereas 9 genes were increased at both timepoints (Fig. 3A). Furthermore, we identified 43 methyltransferases that were downregulated after acute exercise (Fig. 3B), including 8 genes that were downregulated at both timepoints.

After the $12 \mathrm{w}$ exercise intervention, a total of 13 methyltransferases were upregulated whereas $55 \mathrm{mRNA}$ transcripts were downregulated (Supplementary Table 4, Fig. 4A). PRDM1, MECOM, ECE2, HNMT, and SETD9 were the top 5 upregulated methyltransferases, whereas METTL21C, SUV39H1, METTL7A, PNMT, and TRMT112 were the top 5 downregulated. After adjustment for the false discovery rate, no methyltransferases were differentially regulated in WAT after $12 \mathrm{w}$. We did not observe group differences in differential expression after acute exercise in SkM or WAT.

\section{Gene ontology biological process enrichment}

Just after acute exercise, the GO enrichment analyses showed that upregulated methyltransferases were associated with cellular response to stress, biosynthetic processes, peptidyl-lysine methylation, and histone-lysine methylation (Fig. 1B). Methyltransferases that were decreased just after exercise were involved in GOs including protein methylation, alkylation, and positive regulation of gene expression (Fig. 1C). After $2 \mathrm{~h}$ rest, significantly enriched biological processes associated with upregulated methyltransferases included RNA and non-coding RNA processing, RNA modification and methylation as well as ribonucleoprotein complex biogenesis. Downregulated methyltransferases after $2 \mathrm{~h}$ rest were associated with regulation of biological quality (Fig. 2B-C). Genes in GO terms are presented in Supplementary Table 5-7.

After the $12 \mathrm{w}$ exercise intervention upregulated methyltransferases were associated with GOs including cell communication and signaling (Fig. 4B). Downregulated methyltransferases after long-term exercise were associated with cellular component biogenesis, mRNA processing, developmental processes, and ribonucleoprotein complex biogenesis (Fig. 4C).

\section{Methionine, homocysteine and the Met/Hcy ratio in response to acute and long-term exercise}

Because methionine is consumed for methyltransferase reactions, we report the response in methionine and total homocysteine to acute and chronic exercise (Supplementary Table 8). In addition, because Met/Hcy has been used as an indicator of methylation capacity 7,8 we further evaluated its response to exercise. During the baseline acute exercise test, methionine decreased by $40 \%$ from mean (SD) 33.9 (5.71) $\mu \mathrm{mol} / \mathrm{L}$ to 27.9 (3.75) directly after exercise and further to 20.8 (3.34) after $2 \mathrm{~h}$ rest. Plasma total homocysteine increased from 12.2 (2.98) $\mu \mathrm{mol} / \mathrm{L}$ to 13.0 (3.04) just after exercise and then approached baseline after $2 \mathrm{~h}$ rest (12.6 [2.90]). The results were similar after the $12 \mathrm{w}$ intervention.

At baseline before the acute exercise test, mean (SD) Met/Hcy was 2.93 (0.83). Met/Hcy decreased to $2.25(0.59)$ just after exercise and further to $1.73(0.49)$ after $2 \mathrm{~h}$ rest (Fig. 5A). After the $12 \mathrm{w}$ exercise 
intervention, mean (SD) Met/Hcy was 3.03 (0.68) (Fig. 5B). The response to acute exercise after the $12 \mathrm{w}$ intervention was similar to the baseline test (Fig. $5 \mathrm{C}$ ).

To further assess whether changes in plasma Met/Hcy ratio may be linked to SkM metabolism of methionine, we investigated whether genes involved in transport and metabolism of methionine were affected by the acute exercise bout. Indeed, both the light and heavy subunit of the large neutral amino acid transporter, LAT1 (SLC7A5 and SLC7A3), were increased after acute exercise (Fig. 5D-G). Expression levels of the enzyme catalyzing the production of SAM, MAT2A, was also similarly increased after acute exercise at both timepoints (Fig. 5H-I). No effects of long-term exercise were observed in SkM or WAT on these genes.

Next we assessed correlations between the Met/Hcy ratio at baseline with methyltransferase transcripts, the strongest correlations were strongest for DMAP1 and BHMT2 in SkM (Spearman's $r=0.51$ and 0.50, both $\mathrm{p}<0.001)$ and METTL 15 in WAT $(r=0.27, p<0.001)$.

\section{Correlation with phenotypical changes}

We assessed correlations between change in methyltransferase transcript levels and the Met/Hcy ratio in response to $12 \mathrm{w}$ of exercise intervention $(\Delta)$ with the $12 \mathrm{w}$ changes in $\mathrm{GIR}(\Delta \mathrm{GIR}), \mathrm{VO}_{2} \max (\Delta \mathrm{VO} 2 \mathrm{max})$, $m$. vastus lateralis cross-section $(\Delta \mathrm{VL})$ and body fat volume $(\triangle \mathrm{BF})$. Correlation coefficients and corresponding $\mathrm{p}$-values for change in the top 10 most positively and negatively differentially expressed genes with $\triangle \mathrm{GIR}, \triangle \mathrm{VO}_{2}$ max, $\triangle \mathrm{VL}$ and $\triangle \mathrm{BF}$ are presented in Supplementary Table 9-12. $\triangle_{12 \mathrm{w}} P R M T 2$ and $\triangle_{12 \mathrm{w}} H N M T$ were strongly and positively associated with $\triangle \mathrm{GIR}(\mathrm{r}=0.69$ and 0.57 , respectively), whereas $\triangle M E T T L 21 C$ and $\triangle S U V 39 H 1$ were inversely associated with $\triangle \mathrm{GIR}(\mathrm{r}=-0.37$ and -0.42 , respectively $)$. In addition, trends for positive associations were observed for $\triangle D N M T 1$ and $\triangle P R D M 1$ with $\triangle \mathrm{GIR}(\mathrm{r}=0.36$ and 0.36 , respectively). Positive associations were observed for $\Delta_{12 \mathrm{w}} P R D M 1$ and $\triangle_{12 \mathrm{w}} P R M T 2$ with $\Delta \mathrm{VO}_{2} \max \left(\mathrm{r}=0.53\right.$ and 0.49 , respectively). For $\triangle \mathrm{VL}$, we observed a positive association with $\triangle_{12 \mathrm{w}} P N M T$ $(r=0.43)$ and a trend for a positive association with $\Delta_{12 \mathrm{w}} P R D M 1(\mathrm{r}=0.35)$. For $\triangle \mathrm{BF}$, a positive association was observed with $\Delta$ Met/Hcy $(r=0.44, p=0.02)$.

\section{Discussion}

We have investigated transcription of methyltransferases and methylation capacity (Met/Hcy ratio) on data from a $12 \mathrm{w}$ training intervention described previously ${ }^{26}$. After acute and long-term exercise, more than 200 genes with known or probable functions in methyltransferase reactions were transcribed in SkM. Differential gene expression analyses of several methyltransferases in response to acute and longterm exercise in SkM demonstrated that these were primarily involved in the biosynthetic processes, histone- and lysine-methylation (just after exercise), RNA processing ( $2 \mathrm{~h}$ after exercise) as well as cellular communication and developmental processes (long-term exercise). The Met/Hcy ratio was reduced in response to acute exercise but increased slightly following long-term exercise. Several methyltransferases 
were associated with improved in insulin sensitivity measured by clamp (GIR) and $\mathrm{VO}_{2}$ max after the longterm intervention.

The most upregulated methyltransferase just after and $2 \mathrm{~h}$ after acute exercise was $N N M T$, which encodes nicotinamide $\mathrm{N}$-methyltransferase, an enzyme catalyzing the $\mathrm{N}$-methylation of the vitamin $\mathrm{B}_{3^{-}}$ derivative nicotinamide to methylnicotinamide. This finding is partly in line with a previous study demonstrating upregulation of $N N M T$ in response to exercise and suggested that methylnicotinamide may be a signal for WAT lipolysis ${ }^{17}$. In addition, NNMT mRNA levels have been shown to be higher in WAT of patients with type 2 diabetes compared to healthy controls ${ }^{27}$. We did not observe altered NNMT trancription in response to long-term exercise in SkM or WAT. Therefore, change in transcription of NNMT was not associated with improved $\mathrm{GIR}, \mathrm{VO}_{2} \mathrm{max}$, muscle hypertrophy or body fat. Thus, our results support that $N N M T$ may be involved in the acute response to exercise but not necessarily phenotypical improvements.

Few other genes remained differentially expressed throughout the recovery period suggesting a potential shift in biological processes requiring methyltransferase activities from just after to $2 \mathrm{~h}$ after exercise. Just after exercise, regulation of several biosynthetic processes as well as peptidyl and histone-lysine methylation were prominent which are important in epigenetic regulation of gene expression which has received considerable attention for its role in SkM adaptations to exercise together with DNA methylation 11-16, 28,29. In particular, DOT1L, PHF1, and SETD2 were present in most of the enriched GOs, all of which are involved in histone methylation and epigenetic regulation according to OMIM. In particular these methyltransferases are involved in histone $\mathrm{H} 3$ methylation, which indeed seems to play a role in the epigenetic regulation of gene expression in response to exercise ${ }^{28}$. E.g. WDR82 is part of at least four methyltransferase complexes ${ }^{30}$ that trimethylates histone $\mathrm{H} 3$ in the lysine 4 position. This particular histone modification occurred in exercising mice ${ }^{31}$ and was linked to upregulated expression of $P G C-1 a$, a crucial regulator of exercise-induced changes in mitochondrial biogenesis and energy metabolism ${ }^{13,32}$. The downregulated GOs just after exercise also included protein methylation, including the three protein arginine methyltransferases, $P R M T 2, P R M T 6$, and $P R M T 7$. In particular, $P R M T 7$ is highly transcribed in SkM ${ }^{19}$ but has not previously been shown to be affected by acute exercise. Notably, PRMTs are also involved in arginine methylation of histones ${ }^{19}$, and the $\mathrm{GO}$ enrichment analyses thus collectively suggest differential methylation of specific histone amino acid residues just after exercise. These results may aid future research in identifying more targeted approaches in studying the role of epigenetic modifications in SkM during exercise ${ }^{29,33,34}$, and we note that differential expression largely involved histone methyltransferases and not DNA methyltransferases.

After $2 \mathrm{~h}$ rest, GO biological processes including histone methylation and epigenetic regulation were no longer enriched. Instead, analyses suggested that SkM methyltransferases involved in RNA and noncoding RNA processing and methylation, as well as ribonucleoprotein complex biogenesis were upregulated. These processes are important for translational regulation in response to exercise. One study showed that high-intensity interval training increased ribosome abundance and subsequent protein 
translation, and suggested that this is one of the adaptive mechanisms in SkM to long-term high-intensity interval training ${ }^{35}$. Another study showed that trained individuals had higher expression of ribosomal proteins than untrained controls ${ }^{36}$. However, an important distinction was that these relationships were assessed after long-term exercise or in a cross-sectional manner, and not after acute exercise bouts. To our knowledge, the qualitative contribution of methyltransferases to these mechanisms is not known in the immediate recovery phase.

After the 12 w exercise intervention, only 11 out of 210 transcripts with methyltransferase activities were increased. The most upregulated methyltransferase was $P R D M 1$, which is involved in $\mathrm{B}$ cell transcriptional repression through histone modifications ${ }^{37}$. The change in PRDM1 was positively correlated to the improvements in $\mathrm{VO}_{2}$ max and tended to be correlated to improvements in GIR and increased $m$. vastus lateralis cross-sectional area. This is in line with a previous GWAS suggesting that a single nucleotide polymorphism in the PRDM1 encoding region was a significant predictor of improved $\mathrm{VO}_{2} \mathrm{max}^{38}$. However, $P R D M 1$ was not present in the enriched GOs including cell communication and signaling. In these GOs, 8 of the 11 upregulated methyltransferases were present and included genes mostly involved in signaling pathways, histone modifications, and epigenetic regulation such as DNMT1, MECOM, PRMT2, and PRMT6. The change in PRMT2 was strongly and positively associated with improved insulin sensitivity and $\mathrm{VO}_{2} \mathrm{max}$ in the present study, which is in contrast to observations in Prmt $^{-/-}$models exhibiting lower fasting blood glucose and an overall beneficial metabolic profile ${ }^{39}$.

The majority of differentially expressed methyltransferases after long-term exercise were downregulated and associated with broad, unspecific GO terms. The most downregulated transcript was METTL21C, an SkM-specific non-histone protein-lysine methyltransferase involved in regulation of myogenesis, muscle function, and protein catabolism ${ }^{40,41}$. Furthermore, METTL21C has been described as important for regulation of ATPase activity and depletion impairs voluntary running in mice ${ }^{41}$. The physiological and clinical relevance of METTL21C in humans remain to be established, but we note that it was present in several of the enriched downregulated GOs after long-term exercise. Moreover, there was a trend for an inverse association between METTL21C and improvements in GIR, suggesting that subjects with a more negative change in METTL21C mRNA levels had more improved GIR. Notably, METTL21C was the second most upregulated gene $2 \mathrm{~h}$ after exercise, whereas it was significantly downregulated after the 12 w exercise intervention. These findings indicate that METTL21C is not only important in the acute and long-term SkM response to exercise, but may also contribute to beneficial phenotypical responses after a long-term exercise intervention.

Because most methyltransferase reactions depend on methionine we evaluated the effects of exercise on the plasma Met/Hcy ratio, which is increasingly used as a plasma indicator of intracellular methylation capacity ${ }^{7,8}$. We observed a $40 \%$ reduction in plasma Met/Hcy ratio in response to the acute exercise indicating that tissue consumption of methionine for methylation reactions are induced by exercise. Importantly, the decrease in Met/Hcy was driven by the reduction in methionine. Animal studies have shown that SAM consumption increases in liver and the endothelium after exercise promoting increased 
plasma concentrations of total homocysteine concentrations ${ }^{24,25}$, but no studies that we are aware of have characterized methionine metabolism in SkM during exercise. The reduced plasma Met/Hcy ratio and increased expression of LAT1 and MAT2A in our study indicate that SkM uptake and metabolism of methionine could be increased after exercise and involved in methyltransferase reactions. One previous study demonstrated an increase in SkM methionine after exercise in both type I and type II SkM fibers ${ }^{42}$ supporting that increased methionine uptake may occur post-exercise in addition to protein degradation. However, some caution should be shown in interpreting these findings considering that the LAT1 membrane transporter is not specific to methionine. In addition, methyltransferases are generally expressed to a large extent in the liver, and thus the Met/Hcy might not be a good marker of methionine metabolism in SkM.

The strengths of this study include the controlled design, supervised acute and long-term exercise regimen, and deep phenotypical data including improvements in oxygen uptake and systemic insulin sensitivity. This allows for detailed descriptions of the SkM and WAT transcriptomic response just after exercise, in the early recovery phase and after long-term exercise training.

There are also some limitations to the study, including that we only have data on the transcriptomic level, making it impossible to infer on changes in protein activity and the regulation of targeted mechanisms. However, the aims of this study were strictly explorative and its purpose to inform future research seeking to unravel the relationship between methyltransferase reactions and exercise further. With regards to plasma markers, future studies focusing on methylation and methylation reactions should monitor essential intermediates and co-factors for these reactions including SAM and S-adenosylhomocysteine, vitamin $\mathrm{B}_{12}$, folate, choline and betaine.

In conclusion, acute exercise and the immediate recovery phase led to differential expression of methyltransferases related to highly variable biological processes including epigenetic modification and RNA processing, whereas long-term changes in expression were related to developmental processes. In addition, the plasma Met/Hcy ratio decreased in response to acute exercise but increased after long-term exercise training. These results suggest that SkM transcriptomic response to exercise may extend beyond DNA methylation and epigenetic regulation to the processing of other macromolecules and phenotypical improvements.

\section{Methods}

\section{Participants}

The study outline, inclusion criteria and main outcomes of the MyoGlu study has been published previously ${ }^{26}$. Briefly, the original study consisted of 22 subjects classified as either dysglycemic or normoglycemic. Due to the exploratory nature of the present study where the main focus was exercise effects and not group differences, we included data from 4 additional subjects that were excluded in the original study bringing the total $\mathrm{n}$ to 26 . Subject characteristics are presented in Supplementary Table 1. 


\section{Ethics, consent and permissions}

The study was approved by the National Regional Committee for Medical and Health Research Ethics North, Troms $\varnothing$, Norway (Ref No. 2011/882). All participants gave written informed consent to participate. The study protocol was in accordance with the declaration of Helsinki.

\section{Diet}

After an overnight fast, participants consumed a standardized meal $90-120$ min before the $\mathrm{VO}_{2}$ max tests (see below). The meal included bread, cheese, jam and one glass of apple juice.

\section{$\mathrm{VO}_{2} \max$}

During acute exercise, $\mathrm{VO}_{2}$ max was measured and the protocol is available elsewhere ${ }^{26}$. Briefly, participants cycled for 1 min followed by a $15 \mathrm{~W}$ increase in workload every $30 \mathrm{sec}$ until exhaustion. Criteria for a successful test were $\mathrm{O}_{2}$ consumption increasing $<0.5 \mathrm{~mL} \cdot \mathrm{kg}^{-1} \cdot \mathrm{min}^{-1}$ over a $30 \mathrm{~W}$ increase in workload, respiratory exchange ratio $>1.10$, and blood lactate $>7.0 \mathrm{mmol} / \mathrm{L}$.

\section{Strength and endurance training}

The $12 \mathrm{w}$ exercise intervention consisted of four hours of intensive training weekly under professional supervision (two whole-body strength training sessions and two spinning bike interval session). Linear progression in workload for both strength and endurance exercises was implemented in the exercise programs $^{26}$.

\section{Hyperinsulinemic euglycemic clamp}

Participants underwent a standardized endurance session three days prior to the clamp tests and were instructed to refrain from physical exercise and alcohol 48 hours before the tests. After an overnight fast, the hyperinsulinemic euglycemic clamp test was performed with an insulin infusion rate of $40 \mathrm{mU} / \mathrm{m}^{2} / \mathrm{min}$ using human insulin (Actrapid®, NovoNordisk, Bagsvaerd, Denmark). The infusion of glucose $200 \mathrm{mg} / \mathrm{mL}$ was continually adjusted to maintain euglycemia at $5.0 \mathrm{mmol} / \mathrm{L}$. The clamp was maintained for a minimum of $150 \mathrm{~min}^{43}$, until at least $30 \mathrm{~min}$ of stable euglycemia was achieved. The coefficient of variation for glucose measurements was $4 \%$ and $5 \%$ for insulin measurements. Insulin sensitivity was reported as steady-state glucose infusion rate (GIR) relative to body weight. Whole blood glucose concentration was measured using the glucose oxidase method (YSI 2300, Yellow Springs, $\mathrm{OH}$, USA). Plasma glucose concentrations were calculated as whole blood glucose x 1.11943 .

\section{MRI}

Total body fat volume and thigh cross-sectional area was measured by MRI. Ankle-to-neck MRI was performed and included a 3D DIXON acquisition to quantify water and lipid fractions as previously described ${ }^{26}$. 


\section{Tissue sampling}

SkM was taken from $m$. vastus lateralis using a modified Bergström procedure; (1) at rest, (2) just after the 45 min acute exercise test, and (3) after $2 \mathrm{~h}$ rest. SkM biopsies were obtained before and after the 12 $\mathrm{w}$ intervention, as described previously ${ }^{26}$. Biopsies were taken from the right leg at baseline and from the left leg after $12 \mathrm{w}$. One participant did not donate biopsies after $2 \mathrm{~h}$ rest. After sterilization, a lidocainebased local anesthetic was injected into the skin and subcutis prior to the procedure. Biopsies were dissected on a cold aluminum plate before freezing to remove blood and other materials. Tissue for RNA isolation was transferred to RNA-later (Qiagen, Hilden, Germany) overnight and the solution was then drained and stored at $-80^{\circ} \mathrm{C}$.

\section{RNA isolation and cDNA synthesis}

Frozen biopsies were pulverized by pestle and mortar cooled with liquid nitrogen. Frozen biopsies added to $1 \mathrm{~mL}$ QIAzol Lysis Reagent (Qiagen, Hilden, Germany), and homogenized using TissueRuptor (Qiagen) twice at full speed for 15 seconds ${ }^{32}$. Total RNA was then isolated from the homogenates using miRNeasy Mini Kit (Qiagen). RNA integrity and concentration were determined using Agilent RNA 6000 Nano Chips on a Bioanalyzer 2100 (Agilent Technologies Inc, Santa Clara, CA). RNA was converted to cDNA using High-Capacity cDNA Reverse Transcription Kit (Applied Biosystems, Foster, CA, USA). The cDNA reaction mixture was then diluted in water and cDNA equivalent of $25 \mathrm{ng}$ RNA used for each sample.

\section{mRNA sequencing}

mRNA sequencing was performed using the Illumina HiSeq 2000 system (San Diego, CA, USA) with multiplex at the Norwegian Sequencing Centre, University of Oslo using Illumina HiSeq RTA (real-time analysis) v1.17.21.3. Reads passing Illumina's recommended parameters were demultiplexed using CASAVA v1.8.2 (Illumina). For pre-alignment quality checks, we used FastQC v0.10.1 (Illumina). Reads alignment was performed using Tophat v2.0.8. Reads were counted by HTseq v0.6.1. The mRNAsequencing procedure was validated against TaqMan RT-PCR and microarrays, as previously described 44

\section{Plasma methionine and homocysteine}

Blood samples were obtained before the bicycle test (baseline and $12 \mathrm{w}$ ), just after and $2 \mathrm{~h}$ after acute exercise. The determination of plasma methionine and homocysteine were measured by HPLC-MS/MS as has been described in previous publications ${ }^{45-47}$. The methionine and homocysteine data in the present manuscript have been published previously ${ }^{20}$, but are important for the presentation of the plasma Met/Hcy ratio, which has not been published previously. The concentrations of methionine and homocysteine are therefore given in the text and the supplementary information.

\section{Identifying genes involved in methyltransferase activity}


An initial search of The Human Protein Atlas ${ }^{48-50}$ with the query "methyltransferase" was performed and yielded 252 results. The list was extracted and validated for functions either as independent methyltransferases or components of methyltransferase complexes against available information on biological processes, molecular functions, and GOs in UniProtKB/Swiss-Prot, Entrez, QuickGO and The Human Gene Database. Five genes from the initial search (C1orf56, SALL3, ESSRG, CNPY3, and NRM) were excluded after validation. Another 37 genes were additionally excluded because they were not annotated. Thus, we proceeded with 210 genes that encoded methyltransferases or components of methyltransferase complexes or methyltransferase binding as provided by UniProtKB and QuickGO.

\section{Statistics}

Differential gene expression for mRNA transcripts was calculated using the DESeq2 package in $\mathrm{R}(\mathrm{v}$. 3.6.0, R for statistical computing, Vienna, Austria) ${ }^{51}$. We controlled for multiple testing in the differential expression data by calculating the false discovery rate (FDR), and the critical q-value was set to 0.10 indicating that we accepted a $10 \%$ FDR. GO enrichment analyses were performed using the topGO package available from the BioConductor environment in R. For the Met/Hcy ratio, trend analyses over time were performed using linear regression. Finally, we performed Spearman correlation analyses for change in gene expression with change $(\Delta)$ in phenotype including GIR and $\mathrm{VO}_{2} \max$. Because these tests were purely exploratory and non-inferential we did not adjust the $\mathrm{p}$-values for the correlational analyses. All plots were made with the ggplot2 package, and all analyses were performed in R.

\section{Declarations}

\section{Acknowledgements}

A special thank you to Egil Johansen, Daniel S. Tangen, Kristoffer K. Jensen, and Hans Kristian Stadheim at the Norwegian School of Sport Sciences for recruiting the participants and being responsible for the exercise interventions and the practical completion of MyoGlu. We thank A. Halsne, G. Vinje, K. E. Jahnsen, A. Heck, B. Nellemann (Department of Endocrinology, Oslo University Hospital), A. R. Enget (Department of Nutrition, University of Oslo), T. I. Gloppen, T. Dalen, H. Moen, M. A. Dahl, G. Grøthe, K. A Krog, Ø. Skattebo, E. N. Rise (Norwegian School of Sport Sciences) and the Norwegian Sequencing Centre.

\section{Authors contributions}

J.J., K.I.B. and C.A.D. designed the initial study; T.O. conceived the idea and drafted the manuscript; T.O., S.L. and C.T. analyzed the data; All authors interpreted the results, critically revised the paper and approved the final version of the manuscript.

\section{Data availability statement}

The data that support the findings of this study are available on reasonable request from the corresponding author. The data are not publicly available due to privacy or ethical restrictions. 
Competing interests

None declared.

\section{Funding}

This study was funded by grants from the University of Oslo; Johan Throne-Holst Foundation; the Norwegian research council (Grant No: 233946); and Helse Sør-Øst RHF.

\section{References}

1. Petrossian, T. C. \& Clarke, S. G. Uncovering the human methyltransferasome. Mol Cell Proteomics 10, M110 000976, doi:10.1074/mcp.M110.000976 (2011).

2. Brosnan, J. T. \& Brosnan, M. E. The sulfur-containing amino acids: an overview. J Nutr. 136, 16361640 https://doi.org/10.1093/jn/136.6.1636S (2006).

3. Kraus, D. et al. Nicotinamide N-methyltransferase knockdown protects against diet-induced obesity. Nature. 508, 258-262 https://doi.org/10.1038/nature13198 (2014).

4. Strand, E. et al. Serum Carnitine Metabolites and Incident Type 2 Diabetes Mellitus in Patients With Suspected Stable Angina Pectoris. J Clin Endocrinol Metab. 103, 1033-1041 https://doi.org/10.1210/jc.2017-02139 (2018).

5. Bjornestad, E. O. et al. Circulating trimethyllysine and risk of acute myocardial infarction in patients with suspected stable coronary heart disease. J Intern Med. https://doi.org/10.1111/joim.13067 (2020).

6. Sommese, L. et al. Novel epigenetic-sensitive clinical challenges both in type 1 and type 2 diabetes. $J$ Diabetes Complications. 32, 1076-1084 https://doi.org/10.1016/j.jdiacomp.2018.08.012 (2018).

7. Calderon-Larranaga, A. et al. Association of Homocysteine, Methionine, and MTHFR 677C > T Polymorphism With Rate of Cardiovascular Multimorbidity Development in Older Adults in Sweden. JAMA Netw Open. 3, e205316 https://doi.org/10.1001/jamanetworkopen.2020.5316 (2020).

8. Hooshmand, B. et al. Association of Methionine to Homocysteine Status With Brain Magnetic Resonance Imaging Measures and Risk of Dementia. JAMA Psychiatry. https://doi.org/10.1001/jamapsychiatry.2019.1694 (2019).

9. Vinknes, K. J. et al. Plasma Sulfur Amino Acids and Risk of Cerebrovascular Diseases: A Nested Case-Control Study in the EPIC-Norfolk Cohort. Stroke. 52, 172-180 https://doi.org/10.1161/STROKEAHA.120.029177 (2021).

10. Christensen, J. F., Simonsen, C. \& Hojman, P. Exercise Training in Cancer Control and Treatment. Compr Physiol. 9, 165-205 https://doi.org/10.1002/cphy.c180016 (2018).

11. Hunter, D. J. et al. Impact of aerobic exercise and fatty acid supplementation on global and genespecific DNA methylation. Epigenetics. 14, 294-309 https://doi.org/10.1080/15592294.2019.1582276 (2019). 
12. Voisin, S., Eynon, N., Yan, X. \& Bishop, D. J. Exercise training and DNA methylation in humans. Acta Physiol (Oxf). 213, 39-59 https://doi.org/10.1111/apha.12414 (2015).

13. Barres, R. et al. Acute exercise remodels promoter methylation in human skeletal muscle. Cell Metab. 15, 405-411 https://doi.org/10.1016/j.cmet.2012.01.001 (2012).

14. Turner, D. C., Seaborne, R. A. \& Sharples, A. P. Comparative Transcriptome and Methylome Analysis in Human Skeletal Muscle Anabolism, Hypertrophy and Epigenetic Memory. Sci Rep. 9, 4251 https://doi.org/10.1038/s41598-019-40787-0 (2019).

15. Seaborne, R. A. et al. Methylome of human skeletal muscle after acute \& chronic resistance exercise training, detraining \& retraining. Sci Data. 5, 180213 https://doi.org/10.1038/sdata.2018.213 (2018).

16. Landen, S. et al. Genetic and epigenetic sex-specific adaptations to endurance exercise. Epigenetics. 14, 523-535 https://doi.org/10.1080/15592294.2019.1603961 (2019).

17. Strom, K. et al. N(1)-methylnicotinamide is a signalling molecule produced in skeletal muscle coordinating energy metabolism. Sci Rep. 8, 3016 https://doi.org/10.1038/s41598-018-21099-1 (2018).

18. Lee, S. et al. Skeletal muscle phosphatidylcholine and phosphatidylethanolamine respond to exercise and influence insulin sensitivity in men. Sci Rep. 8, 6531 https://doi.org/10.1038/s41598-018-24976$x$ (2018).

19. vanLieshout, T. L., Bonafiglia, J. T., Gurd, B. J. \& Ljubicic, V. Protein arginine methyltransferase biology in humans during acute and chronic skeletal muscle plasticity. J Appl Physiol (1985). 127, 867-880 https://doi.org/10.1152/japplphysiol.00142.2019 (2019).

20. Lee, S. et al. Plasma Sulphur-Containing Amino Acids, Physical Exercise and Insulin Sensitivity in Overweight Dysglycemic and Normal Weight Normoglycemic Men. Nutrients. 11, https://doi.org/10.3390/nu11010010 (2018).

21. Olsen, T. et al. Exhaustive Exercise and Post-exercise Protein Plus Carbohydrate Supplementation Affect Plasma and Urine Concentrations of Sulfur Amino Acids, the Ratio of Methionine to Homocysteine and Glutathione in Elite Male Cyclists. Front Physiol. 11, 609335 https://doi.org/10.3389/fphys.2020.609335 (2020).

22. Mentch, S. J. et al. Histone Methylation Dynamics and Gene Regulation Occur through the Sensing of One-Carbon Metabolism. Cell Metab. 22, 861-873 https://doi.org/10.1016/j.cmet.2015.08.024 (2015).

23. Shiraki, N. et al. Methionine metabolism regulates maintenance and differentiation of human pluripotent stem cells. Cell Metab. 19, 780-794 https://doi.org/10.1016/j.cmet.2014.03.017 (2014).

24. Riberio, D. F., Cella, P. S., da Silva, L., Jordao, A. A. \& Deminice, R. Acute exercise alters homocysteine plasma concentration in an intensity-dependent manner due increased methyl flux in liver of rats. Life Sci. 196, 63-68 https://doi.org/10.1016/j.Ifs.2018.01.003 (2018).

25. Zhang, Y. et al. Chronic exercise mediates epigenetic suppression of L-type Ca2 + channel and BKCa channel in mesenteric arteries of hypertensive rats. $J$ Hypertens. https://doi.org/10.1097/HJH.0000000000002457 (2020). 
26. Langleite, T. M. et al. Insulin sensitivity, body composition and adipose depots following $12 \mathrm{w}$ combined endurance and strength training in dysglycemic and normoglycemic sedentary men. Arch Physiol Biochem. 122, 167-179 https://doi.org/10.1080/13813455.2016.1202985 (2016).

27. Kannt, A. et al. Association of nicotinamide-N-methyltransferase mRNA expression in human adipose tissue and the plasma concentration of its product, 1-methylnicotinamide, with insulin resistance. Diabetologia. 58, 799-808 https://doi.org/10.1007/s00125-014-3490-7 (2015).

28. Dimauro, I., Paronetto, M. P. \& Caporossi, D. Exercise, redox homeostasis and the epigenetic landscape. Redox Biol. 101477, https://doi.org/10.1016/j.redox.2020.101477 (2020).

29. Beiter, T., Niess, A. M. \& Moser, D. Transcriptional memory in skeletal muscle. Don't forget (to) exercise. J Cell Physiol. 235, 5476-5489 https://doi.org/10.1002/jcp.29535 (2020).

30. Lee, J. H., You, J., Dobrota, E. \& Skalnik, D. G. Identification and characterization of a novel human PP1 phosphatase complex. J Biol Chem. 285, 24466-24476 https://doi.org/10.1074/jbc.M110.109801 (2010).

31. Lochmann, T. L., Thomas, R. R., Bennett, J. P. Jr. \& Taylor, S. M. Epigenetic Modifications of the PGC1alpha Promoter during Exercise Induced Expression in Mice. PLoS One. 10, e0129647 https://doi.org/10.1371/journal.pone.0129647 (2015).

32. Norheim, F. et al. The effects of acute and chronic exercise on PGC-1alpha, irisin and browning of subcutaneous adipose tissue in humans. FEBS J. 281, 739-749 https://doi.org/10.1111/febs.12619 (2014).

33. Dimauro, I., Paronetto, M. P. \& Caporossi, D. Exercise, redox homeostasis and the epigenetic landscape. Redox Biol. 35, 101477 https://doi.org/10.1016/j.redox.2020.101477 (2020).

34. Widmann, M., Niess, A. M. \& Munz, B. Physical Exercise and Epigenetic Modifications in Skeletal Muscle. Sports Med. 49, 509-523 https://doi.org/10.1007/s40279-019-01070-4 (2019).

35. Robinson, M. M. et al. Enhanced Protein Translation Underlies Improved Metabolic and Physical Adaptations to Different Exercise Training Modes in Young and Old Humans. Cell Metab. 25, 581592 https://doi.org/10.1016/j.cmet.2017.02.009 (2017).

36. Liu, D. et al. Immune adaptation to chronic intense exercise training: new microarray evidence. $B M C$ Genomics. 18, 29 https://doi.org/10.1186/s12864-016-3388-5 (2017).

37. Di Zazzo, E., De Rosa, C., Abbondanza, C. \& Moncharmont, B. P. R. D. M. Proteins: Molecular Mechanisms in Signal Transduction and Transcriptional Regulation. Biology (Basel). 2, 107-141 https://doi.org/10.3390/biology2010107 (2013).

38. Bouchard, C. et al. Genomic predictors of the maximal O(2) uptake response to standardized exercise training programs. J Appl Physiol (1985). 110, 1160-1170 https://doi.org/10.1152/japplphysiol.00973.2010 (2011).

39. Iwasaki, H. et al. Disruption of protein arginine $\mathrm{N}$-methyltransferase 2 regulates leptin signaling and produces leanness in vivo through loss of STAT3 methylation. Circ Res. 107, 992-1001 https://doi.org/10.1161/CIRCRESAHA.110.225326 (2010). 
40. Wang, C. et al. Methyltransferase-like 21c methylates and stabilizes the heat shock protein Hspa8 in type I myofibers in mice. J Biol Chem. 294, 13718-13728 https://doi.org/10.1074/jbc.RA119.008430 (2019).

41. Wiederstein, J. L. et al. Skeletal Muscle-Specific Methyltransferase METTL21C Trimethylates p97 and Regulates Autophagy-Associated Protein Breakdown. Cell Rep. 23, 1342-1356 https://doi.org/10.1016/j.celrep.2018.03.136 (2018).

42. Essen-Gustavsson, B. \& Blomstrand, E. Effect of exercise on concentrations of free amino acids in pools of type I and type II fibres in human muscle with reduced glycogen stores. Acta Physiol Scand. 174, 275-281 https://doi.org/10.1046/j.1365-201x.2002.00942.x (2002).

43. DeFronzo, R. A., Tobin, J. D. \& Andres, R. Glucose clamp technique: a method for quantifying insulin secretion and resistance. The American journal of physiology. 237, E214-223 https://doi.org/10.1152/ajpendo.1979.237.3.E214 (1979).

44. Lee, S. et al. Effects of long-term exercise on plasma adipokine levels and inflammation-related gene expression in subcutaneous adipose tissue in sedentary dysglycaemic, overweight men and sedentary normoglycaemic men of healthy weight. Diabetologia. 62, 1048-1064 https://doi.org/10.1007/s00125-019-4866-5 (2019).

45. Olsen, T. et al. Effects of dietary methionine and cysteine restriction on plasma biomarkers, serum fibroblast growth factor 21, and adipose tissue gene expression in women with overweight or obesity: a double-blind randomized controlled pilot study. J Trans/ Med. 18, 122 https://doi.org/10.1186/s12967-020-02288-x (2020).

46. Olsen, T. et al. Combining Dietary Sulfur Amino Acid Restriction with Polyunsaturated Fatty Acid Intake in Humans: A Randomized Controlled Pilot Trial. Nutrients. 10, https://doi.org/10.3390/nu10121822 (2018).

47. Antoniades, C. et al. MTHFR $677 \mathrm{C}>$ T Polymorphism reveals functional importance for 5methyltetrahydrofolate, not homocysteine, in regulation of vascular redox state and endothelial function in human atherosclerosis. Circulation. 119, 2507-2515 https://doi.org/10.1161/CIRCULATIONAHA.108.808675 (2009).

48. Uhlen, M. et al. Proteomics. Tissue-based map of the human proteome. Science. 347, 1260419 https://doi.org/10.1126/science.1260419 (2015).

49. Thul, P. J. et al. A subcellular map of the human proteome. Science. 356, https://doi.org/10.1126/science.aal3321 (2017).

50. Uhlen, M. et al. A pathology atlas of the human cancer transcriptome. Science. 357, https://doi.org/10.1126/science.aan2507 (2017).

51. Anders, S. \& Huber, W. Differential expression analysis for sequence count data. Genome biology. 11, R106 https://doi.org/10.1186/gb-2010-11-10-r106 (2010).

\section{Figures}




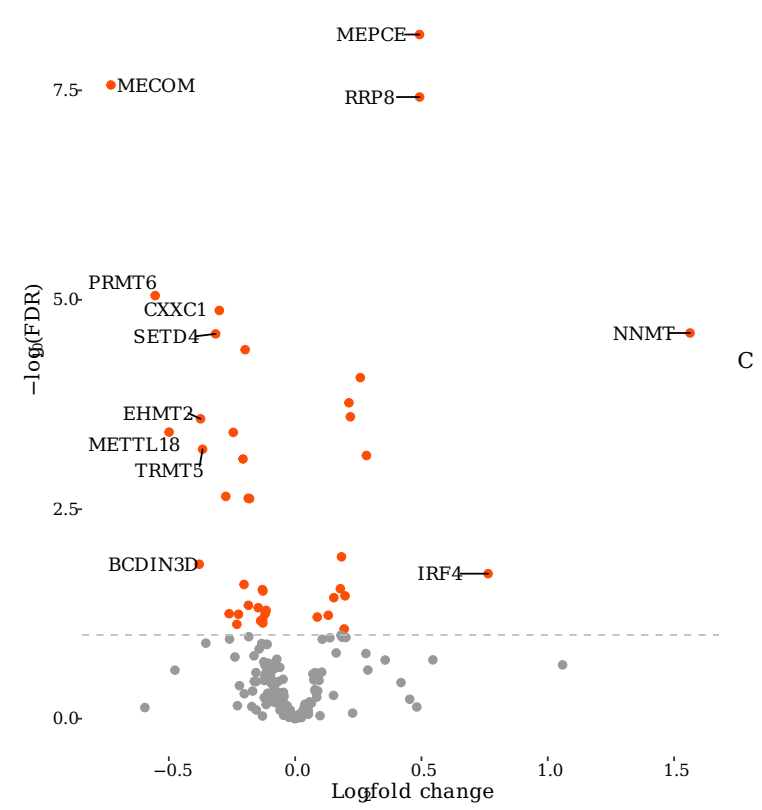

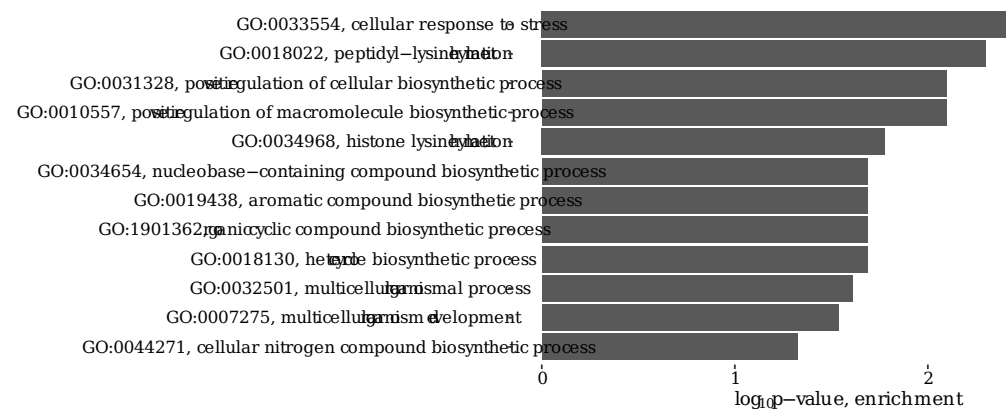

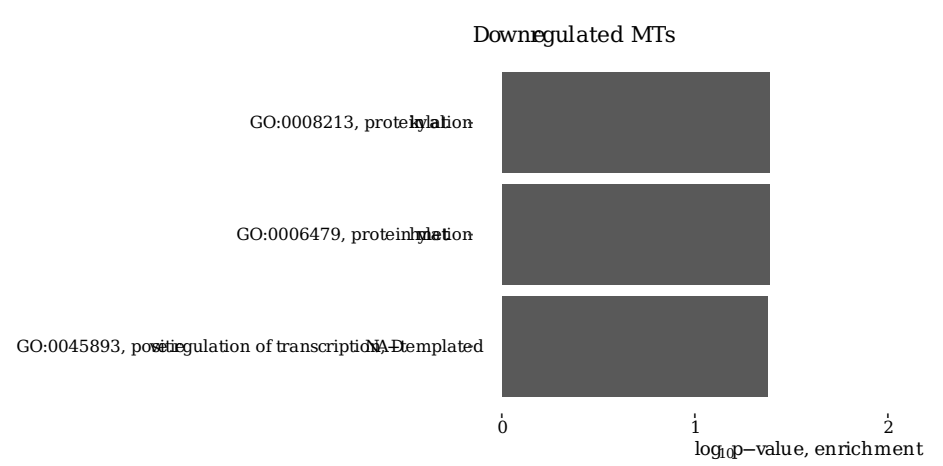

\section{Figure 1}

A) Differential expression (just after vs. before exercise) of methyltransferases. Genes with log2 fold change $<-0.3$ or $>0.3$ and with $q<0.1$ are annotated. Red color indicates significant differential expression after controlling for the FDR. B) Gene enrichment analysis for upregulated methyltransferases.

C) Gene enrichment analysis for downregulated methyltransferases. Abbreviation; MT, methyltransferase

A

$15-$

$\bullet H D A C 9$

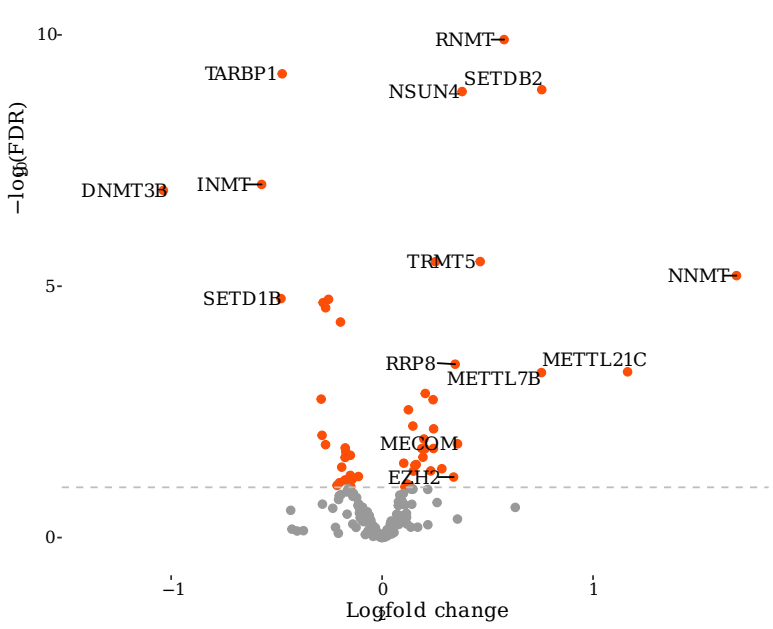

B

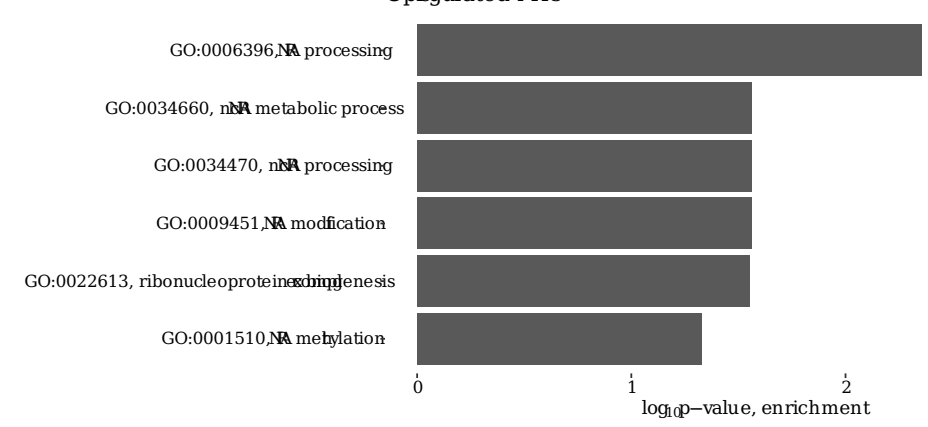

C

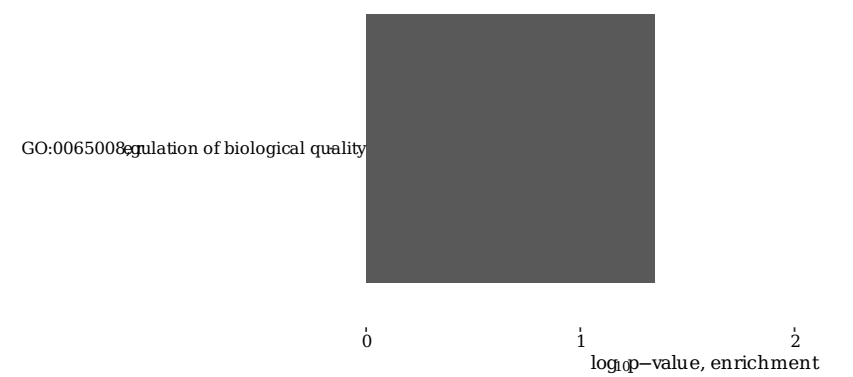


A) Differential expression ( $2 \mathrm{~h}$ after vs. before exercise) of methyltransferases. Genes with log2 fold change $<-0.3$ or $>0.3$ and with $q<0.1$ are annotated. Red color indicates significant differential expression after controlling for the FDR B) Gene enrichment analysis for upregulated methyltransferases. C) Gene enrichment analysis for downregulated methyltransferases. Abbreviation; MT, methyltransferase A

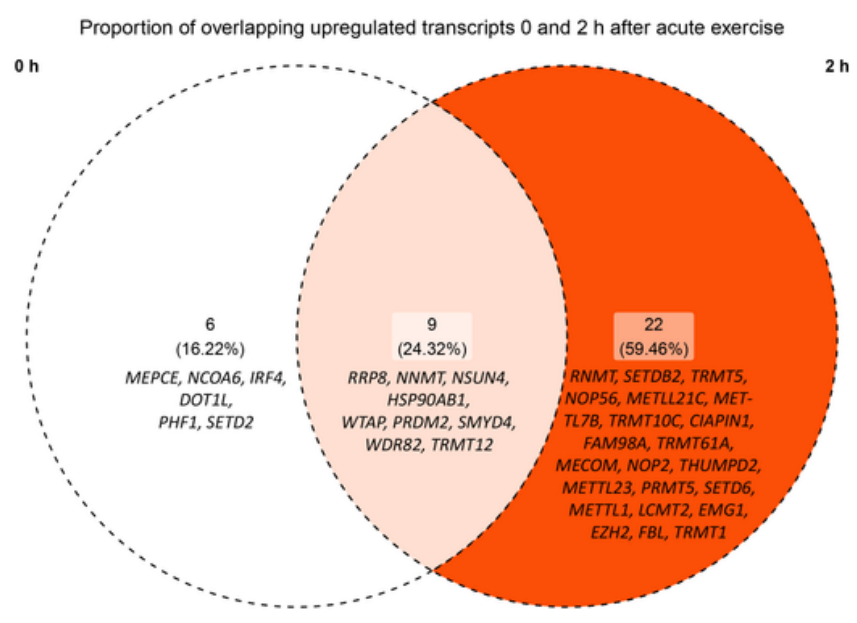

B

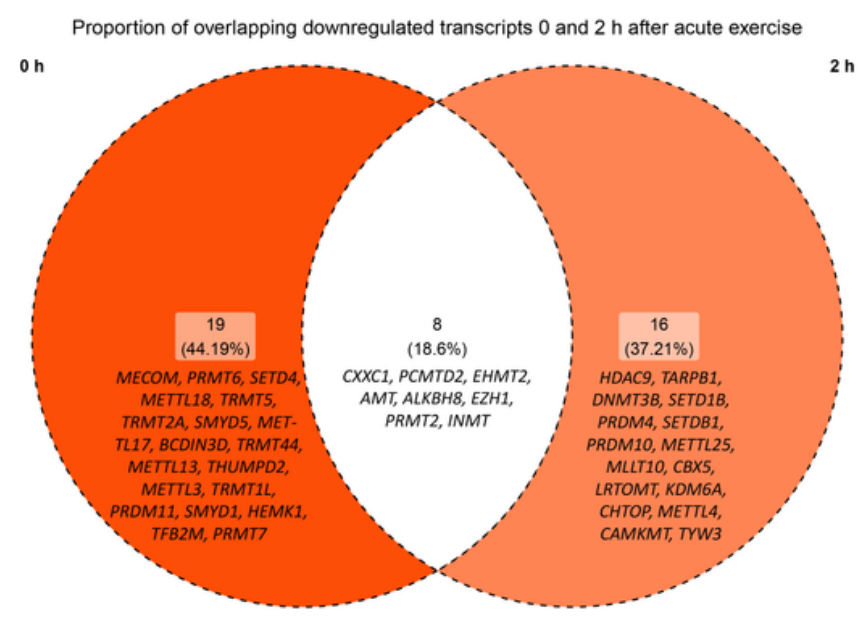

\section{Figure 3}

A) Shows uniquely and intersecting upregulated methyltransferase transcripts for just and $2 \mathrm{~h}$ after acute exercise. B) Shows uniquely and intersecting downregulated methyltransferase transcripts for just and 2 $\mathrm{h}$ after acute exercise. Colour gradient indicates condition with highest proportion of unique transcripts.

A

8 -

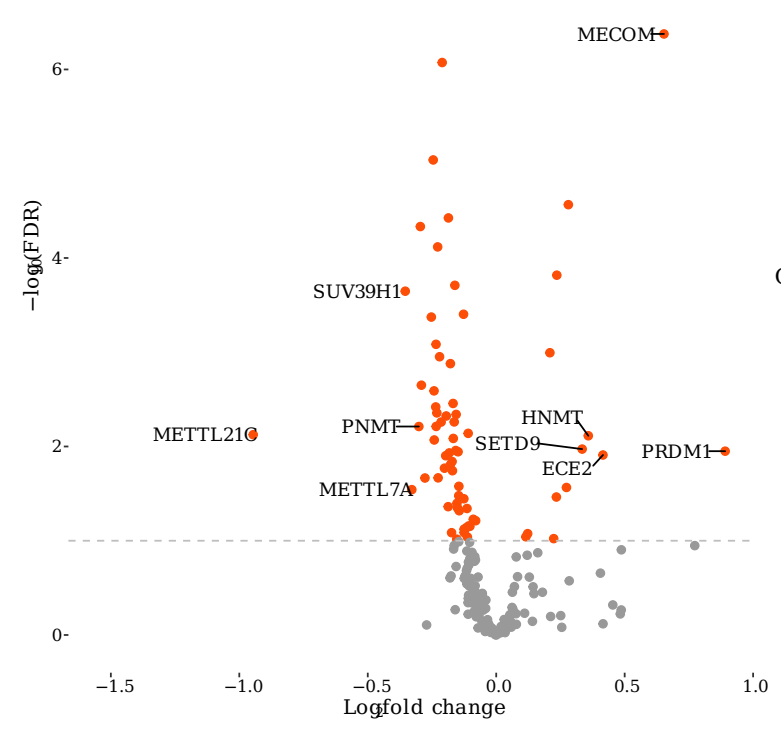

B

C

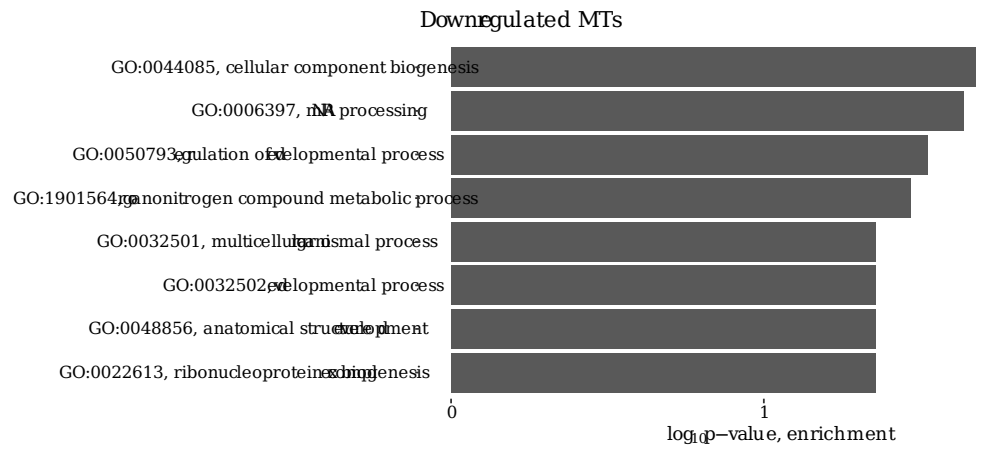

i

\section{Figure 4}


A) Differential expression (12 w vs. baseline) of methyltransferases. Genes with log2 fold change $<-0.3$ or $>0.3$ and with $\mathrm{q}<0.1$ are annotated. Red color indicates significant differential expression after controlling for the FDR B) Gene enrichment analysis for upregulated methyltransferases. C) Gene enrichment analysis for downregulated methyltransferases. Abbreviation; MT, methyltransferase

A

Acute exercise bout, baseline
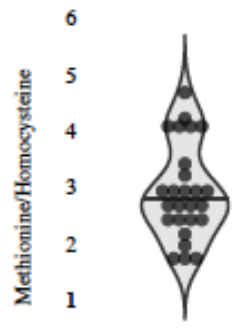

Anival

Just after exercise $2 \mathrm{~h}$ recovery

D

Acute exercise bout, baseline

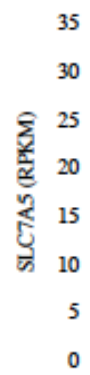

F
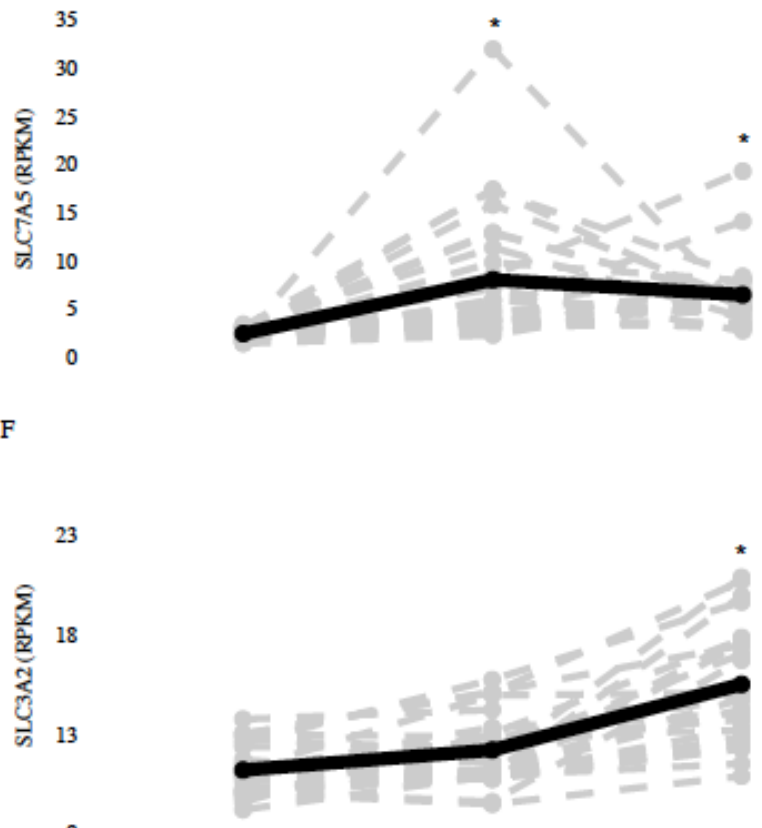

$\mathrm{H}$

8
B

12 w exercise intervention

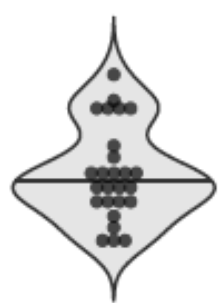

Baseline

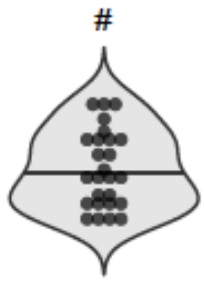

12 weeks
C Acute exercise bout, post-intervention

E Acute exercise bout, post-intervention
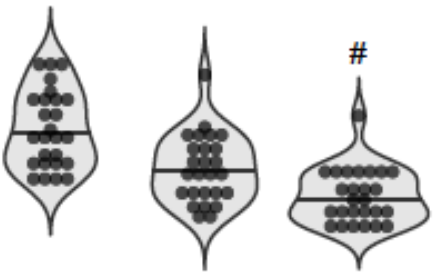

Arrival Just after exercise $2 \mathrm{~h}$ recovery

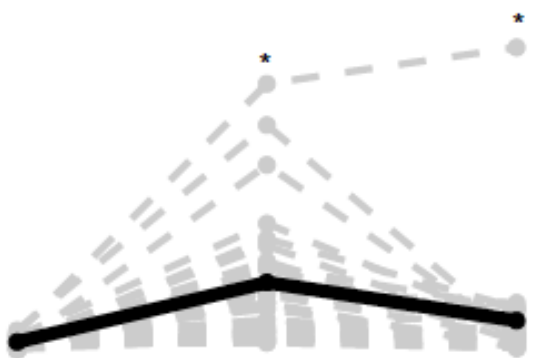

G

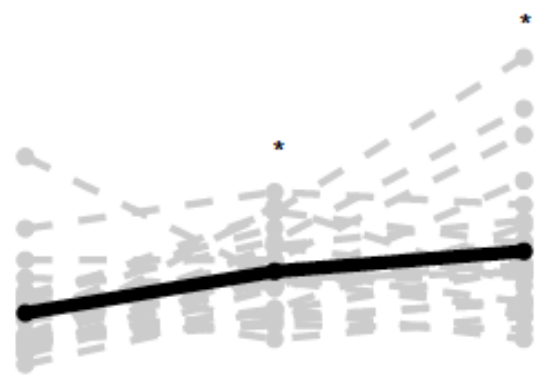

I

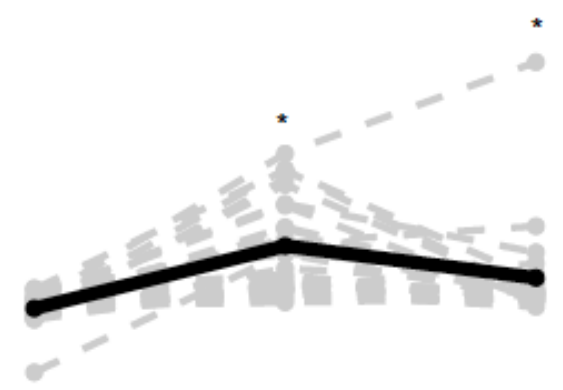

Arrival

Just after exercise

$2 \mathrm{~h}$ recovery

Arrival

Just after exercise

$2 \mathrm{~h}$ recovery

Figure 5 
Change in the ratio of plasma methionine to total homocysteine, an indicator of methylation capacity after acute exercise at baseline (A), $12 \mathrm{w}$ (B) and acute exercise after $12 \mathrm{w}$ (C). D) SLC7A5 response to acute exercise at baseline and E) after the intervention. F) SLC3A2 response to acute exercise at baseline and $\mathrm{G}$ ) post-intervention. $\mathrm{H}$ ) MAT2A response to exercise at baseline and I) after the intervention. \# = ptrend $<0.001 ;{ }^{*}=p<0.05$

\section{Supplementary Files}

This is a list of supplementary files associated with this preprint. Click to download.

- Supplementaryinformation.pdf 